\title{
A BAILEY TREE FOR INTEGRALS
}

\author{
V.P. SPIRIDONOV
}

\begin{abstract}
The notion of integral Bailey pairs is introduced. Using the single variable elliptic beta integral, we construct an infinite binary tree of identities for elliptic hypergeometric integrals. Two particular sequences of identities are explicitly described.
\end{abstract}

Series and integrals of hypergeometric type have many applications in mathematical physics. Investigation of the corresponding special functions can therefore be relevant for various computations in theoretical models of reality. Bailey chains provide powerful tools for generating infinite sequences of summation or transformation formulas for hypergeometric type series. For a review of the corresponding formalism and many $q$-series identities obtained with its help, see, e.g., 1], 2]. Recently, we extended the scope of applications of this technique to elliptic hypergeometric series [3. More precisely, an elliptic generalization of the Andrews' well-poised Bailey chain [1] has been found and its simplest consequences have been analyzed. This lifted also one of the Andrews-Berkovich identities 4 to the elliptic level.

During the work on [3], we came to a principal conclusion that there must exist Bailey chains for integrals, but the first attempts to build a simple example of such a chain were not successful. In [5], a Bailey type symmetry transformation was constructed for a pair of elliptic hypergeometric integrals. This result gives the tools necessary for an appropriate generalization of the elliptic Bailey chain technique of 3. In the present note, we construct two examples of Bailey chains for integrals directly at the level of the most complicated known type of beta integrals of one variable, namely, the elliptic beta integral of 6 . Being used together, they form a binary tree of identities for elliptic hypergeometric integrals.

We denote by $\mathbb{T}$ the positively oriented unit circle and take two base variables $q, p$, $|q|,|p|<1$, and five complex parameters $t_{m}, m=0, \ldots, 4$, satisfying the inequalities $\left|t_{m}\right|<1,|p q|<|A|$, where $A \equiv \prod_{r=0}^{4} t_{r}$. Then the elliptic beta integral

$$
\mathcal{N}_{E}(\mathbf{t})=\frac{1}{2 \pi i} \int_{\mathbb{T}} \frac{\prod_{m=0}^{4} \Gamma\left(z^{ \pm} t_{m}\right)}{\Gamma\left(z^{ \pm 2}, z^{ \pm} A\right)} \frac{d z}{z},
$$

admits the exact evaluation [6]:

$$
\mathcal{N}_{E}(\mathbf{t})=\frac{2 \prod_{0 \leq m<s \leq 4} \Gamma\left(t_{m} t_{s}\right)}{(q ; q)_{\infty}(p ; p)_{\infty} \prod_{m=0}^{4} \Gamma\left(A t_{m}^{-1}\right)} .
$$

Date: May 2003; to be published in Theor. Math. Phys. (Russian). 
The standard notation $(q ; q)_{\infty}=\prod_{n=1}^{\infty}\left(1-q^{n}\right)$ and the following conventions are implied throughout the paper:

$$
\begin{aligned}
& \Gamma\left(t z^{ \pm}\right)=\Gamma\left(t z, t z^{-1}\right), \quad \Gamma\left(z^{ \pm 2}\right)=\Gamma\left(z^{2}, z^{-2}\right), \\
& \Gamma\left(t z^{ \pm} x^{ \pm}\right)=\Gamma\left(t z x, t z x^{-1}, t z^{-1} x, t z^{-1} x^{-1}\right), \\
& \Gamma\left(t_{1}, \ldots, t_{m}\right) \equiv \prod_{r=1}^{m} \Gamma\left(t_{r} ; q, p\right),
\end{aligned}
$$

where $\Gamma(z ; q, p)$ is the elliptic gamma function

$$
\Gamma(z ; q, p)=\prod_{j, k=0}^{\infty} \frac{1-z^{-1} q^{j+1} p^{k+1}}{1-z q^{j} p^{k}}
$$

(for a brief historical account of this function and relevant references, see, e.g., [5]). For $p=0$, formula (11) is reduced to the Nassrallah-Rahman $q$-beta integral which can be reduced further to the Askey-Wilson integral [7.

The main definition of the present paper introduces the notion of Bailey pairs for integrals.

Definition. Two functions $\alpha(z, t)$ and $\beta(z, t), z, t \in \mathbb{C}$, are said to form an integral elliptic Bailey pair with respect to the parameter $t$ if they satisfy the following relation

$$
\beta(w, t)=\kappa \int_{\mathbb{T}} \Gamma\left(t w^{ \pm} z^{ \pm}\right) \alpha(z, t) \frac{d z}{z}, \quad \kappa \equiv \frac{(p ; p)_{\infty}(q ; q)_{\infty}}{4 \pi i} .
$$

Suppose that we have found a particular integral elliptic Bailey pair. Then there exists a possibility to build an infinite sequence of such pairs out of the given one.

Theorem 1. Whenever two functions $\alpha(z, t)$ and $\beta(z, t)$ form an integral elliptic Bailey pair with respect to $t$, the new functions defined for $w \in \mathbb{T}$ as

$$
\alpha^{\prime}(w, s t)=\frac{\Gamma\left(t u w^{ \pm}\right)}{\Gamma\left(t s^{2} u w^{ \pm}\right)} \alpha(w, t)
$$

and

$$
\beta^{\prime}(w, s t)=\kappa \frac{\Gamma\left(t^{2} s^{2}, t^{2} s u w^{ \pm}\right)}{\Gamma\left(s^{2}, t^{2}, s u w^{ \pm}\right)} \int_{\mathbb{T}} \frac{\Gamma\left(s w^{ \pm} x^{ \pm}, u x^{ \pm}\right)}{\Gamma\left(x^{ \pm 2}, t^{2} s^{2} u x^{ \pm}\right)} \beta(x, t) \frac{d x}{x}
$$

form an integral elliptic Bailey pair with respect to the parameter st. Here $t, s, u$ are arbitrary complex parameters satisfying the constraints $|t|,|s|,|u|<1,|p q|<\left|t^{2} s^{2} u\right|$.

Proof. We substitute definition (4) into relation (6)

$$
\begin{aligned}
\beta^{\prime}(w, s t)= & \kappa^{2} \frac{\Gamma\left(t^{2} s^{2}, t^{2} s u w^{ \pm}\right)}{\Gamma\left(s^{2}, t^{2}, s u w^{ \pm}\right)} \\
& \times \int_{\mathbb{T}^{2}} \frac{\Gamma\left(s w^{ \pm} x^{ \pm}, t z^{ \pm} x^{ \pm}, u x^{ \pm}\right)}{\Gamma\left(x^{ \pm 2}, t^{2} s^{2} u x^{ \pm}\right)} \alpha(z, t) \frac{d z}{z} \frac{d x}{x} .
\end{aligned}
$$

Since the integrands are bounded on $\mathbb{T}$, we may change in this expression the order of integrations (i.e., integrate first with respect to $x$ ) and apply formula (11). This yields

$$
\begin{aligned}
\beta^{\prime}(w, s t) & =\kappa \int_{\mathbb{T}} \frac{\Gamma\left(s t w^{ \pm} z^{ \pm}, t u z^{ \pm}\right)}{\Gamma\left(t s^{2} u z^{ \pm}\right)} \alpha(z, t) \frac{d z}{z} \\
& =\kappa \int_{\mathbb{T}} \Gamma\left(s t w^{ \pm} z^{ \pm}\right) \alpha^{\prime}(z, s t) \frac{d z}{z}
\end{aligned}
$$


that is the functions $\alpha^{\prime}(w, s t)$ and $\beta^{\prime}(w, s t)$ form an integral Bailey pair with respect to the parameter st.

In some sense, this theorem may be called as an integral Bailey lemma.

If we substitute into integral (11) relations $t_{3}=t w, t_{4}=t w^{-1}$, then it is not difficult to figure out an initial integral elliptic Bailey pair:

$$
\begin{aligned}
& \alpha(z, t)=\frac{\prod_{r=0}^{2} \Gamma\left(t_{r} z^{ \pm}\right)}{\Gamma\left(z^{ \pm 2}, t^{2} t_{0} t_{1} t_{2} z^{ \pm}\right)}, \\
& \beta(w, t)=\Gamma\left(t^{2}\right) \prod_{0 \leq r<j \leq 2} \frac{\Gamma\left(t_{r} t_{j}\right)}{\Gamma\left(t^{2} t_{r} t_{j}\right)} \frac{\prod_{r=0}^{2} \Gamma\left(t t_{r} w^{ \pm}\right)}{\Gamma\left(t t_{0} t_{1} t_{2} w^{ \pm}\right)},
\end{aligned}
$$

where $|t|,\left|t_{r}\right|<1,|p q|<\left|t^{2} t_{0} t_{1} t_{2}\right|$. Substituting these expressions into chain rules (5) and (6), we obtain

$$
\begin{aligned}
\alpha^{\prime}(z, s t)= & \frac{\Gamma\left(t u z^{ \pm}\right) \prod_{r=0}^{2} \Gamma\left(t_{r} z^{ \pm}\right)}{\Gamma\left(t s^{2} u z^{ \pm}, z^{ \pm 2}, t^{2} t_{0} t_{1} t_{2} z^{ \pm}\right)}, \\
\beta^{\prime}(w, s t)= & \kappa \frac{\Gamma\left(t^{2} s^{2}, t^{2} s u w^{ \pm}\right)}{\Gamma\left(s^{2}, s u w^{ \pm}\right)} \prod_{0 \leq r<j \leq 2} \frac{\Gamma\left(t_{r} t_{j}\right)}{\Gamma\left(t^{2} t_{r} t_{j}\right)} \\
& \times \int_{\mathbb{T}} \frac{\Gamma\left(s w^{ \pm} x^{ \pm}, u x^{ \pm}\right) \prod_{r=0}^{2} \Gamma\left(t t_{r} x^{ \pm}\right)}{\Gamma\left(x^{ \pm 2}, t^{2} s^{2} u x^{ \pm}, t t_{0} t_{1} t_{2} x^{ \pm}\right)} \frac{d x}{x} .
\end{aligned}
$$

Being plugged into key generating relation (8), these functions yield a symmetry transformation for two elliptic hypergeometric integrals:

$$
\begin{aligned}
\prod_{j=0}^{2} & \frac{\Gamma\left(B t_{j}^{-1}\right)}{\Gamma\left(t^{2} B t_{j}^{-1}\right)} \int_{\mathbb{T}} \frac{\prod_{j=1}^{3} \Gamma\left(t t_{j} z^{ \pm}, s_{j} z^{ \pm}\right)}{\Gamma\left(z^{ \pm 2}, t^{2} S z^{ \pm}, t B z^{ \pm}\right)} \frac{d z}{z} \\
& =\prod_{j=0}^{2} \frac{\Gamma\left(S s_{j}^{-1}\right)}{\Gamma\left(t^{2} S s_{j}^{-1}\right)} \int_{\mathbb{T}} \frac{\prod_{j=1}^{3} \Gamma\left(t s_{j} z^{ \pm}, t_{j} z^{ \pm}\right)}{\Gamma\left(z^{ \pm 2}, t^{2} B z^{ \pm}, t S z^{ \pm}\right)} \frac{d z}{z},
\end{aligned}
$$

where $s_{0}=u, s_{1}=s w, s_{2}=s w^{-1}$ and $B=\prod_{j=0}^{2} t_{j}, S=\prod_{j=0}^{2} s_{j}$. Identity (11) was established in [5] and the integral elliptic Bailey chain (40)-([6) is constructed with the help of a generalization of the corresponding method of derivation of this equality.

Iterative application of rules (5) and (6) generates an infinite chain of identities for elliptic hypergeometric integrals. The result of $m$-fold iteration has the form

$$
\begin{aligned}
& \alpha^{(m)}\left(x, \prod_{l=1}^{m} s_{l} t\right)=\prod_{k=1}^{m} \frac{\Gamma\left(t \prod_{l=1}^{k-1} s_{l} u_{k} x^{ \pm}\right)}{\Gamma\left(t \prod_{l=1}^{k-1} s_{l} s_{k}^{2} u_{k} x^{ \pm}\right)} \alpha^{(0)}(x, t) \\
& \beta^{(m)}\left(x_{m+1}, \prod_{l=1}^{m} s_{l} t\right)=\kappa^{m} \prod_{k=1}^{m} \frac{\Gamma\left(t^{2} \prod_{l=1}^{k} s_{l}^{2}\right)}{\Gamma\left(s_{k}^{2}, t^{2} \prod_{l=1}^{k-1} s_{l}^{2}\right)} \\
& \quad \times \int_{\mathbb{T}^{m}} \prod_{k=1}^{m} \frac{\Gamma\left(t^{2} \prod_{l=1}^{k-1} s_{l}^{2} s_{k} u_{k} x_{k+1}^{ \pm}, s_{k} x_{k+1}^{ \pm} x_{k}^{ \pm}, u_{k} x_{k}^{ \pm}\right)}{\Gamma\left(s_{k} u_{k} x_{k+1}^{ \pm}, x_{k}^{ \pm 2}, t^{2} \prod_{l=1}^{k} s_{l}^{2} u_{k} x_{k}^{ \pm}\right)} \beta^{(0)}\left(x_{1}, t\right) \prod_{l=1}^{m} \frac{d x_{l}}{x_{l}}
\end{aligned}
$$


Using (9) and (10) as the initial functions $\alpha^{(0)}(x, t)$ and $\beta^{(0)}(x, t)$ and substituting (12) and (13) into (4), we obtain the identity

$$
\begin{aligned}
\kappa^{m-1} \prod_{k=1}^{m} & \frac{\Gamma\left(t^{2} \prod_{l=1}^{k} s_{l}^{2}\right)}{\Gamma\left(s_{k}^{2}, t^{2} \prod_{l=1}^{k-1} s_{l}^{2}\right)} \int_{\mathbb{T}^{m}} \frac{\prod_{r=0}^{2} \Gamma\left(t t_{r} x_{1}^{ \pm}\right)}{\Gamma\left(t t_{0} t_{1} t_{2} x_{1}^{ \pm}\right)} \\
& \times \prod_{k=1}^{m} \frac{\Gamma\left(t^{2} \prod_{l=1}^{k-1} s_{l}^{2} s_{k} u_{k} x_{k+1}^{ \pm}, s_{k} x_{k+1}^{ \pm} x_{k}^{ \pm}, u_{k} x_{k}^{ \pm}\right)}{\Gamma\left(s_{k} u_{k} x_{k+1}^{ \pm}, x_{k}^{ \pm 2}, t^{2} \prod_{l=1}^{k} s_{l}^{2} u_{k} x_{k}^{ \pm}\right)} \frac{d x_{l}}{x_{l}} \\
= & \frac{1}{\Gamma\left(t^{2}\right)} \prod_{0 \leq r<j \leq 2} \frac{\Gamma\left(t^{2} t_{r} t_{j}\right)}{\Gamma\left(t_{r} t_{j}\right)} \int_{\mathbb{T}} \frac{\Gamma\left(t \prod_{l=1}^{m} s_{l} x_{m+1}^{ \pm} x^{ \pm}\right) \prod_{r=0}^{2} \Gamma\left(t_{r} x^{ \pm}\right)}{\Gamma\left(x^{ \pm 2}, t^{2} t_{0} t_{1} t_{2} x^{ \pm}\right)} \\
& \times \prod_{k=1}^{m} \frac{\Gamma\left(t \prod_{l=1}^{k-1} s_{l} u_{k} x^{ \pm}\right)}{\Gamma\left(t \prod_{l=1}^{k-1} s_{l} s_{k}^{2} u_{k} x^{ \pm}\right)} \frac{d x}{x},
\end{aligned}
$$

which can be rewritten as (11) for $m=1$. In some sense, relation (14) is a high level integral generalization of the multiple series Rogers-Ramanujan type identities derived in 8. Integral analogues of some of the Rogers-Ramanujan identities have been considered in 9]. Taking into account a great deal of activity around Bailey chains for series, it is surprising that their integral analogues did not get appropriate attention in the literature. Some examples of the one step Bailey type transformations for integrals were known for a long time. Actually, all the changes of the orders of integrations in multiple integrals used in [10, 11, 12, 13, 14, [5] may be interpreted as integral generalizations of the Bailey's change of the order of summations for series 1], 2]. The general idea of using a change of the integrations order for obtaining useful information about integrals goes back to Poisson's and Jacobi's methods of derivation of the ordinary beta integral [15].

It is known that for a taken definition of Bailey pairs there sometimes exist several different chain substitution rules (or Bailey lemmas). In this case one gets a more complicated structure of the derived set of identities, which is called as the Bailey lattice [16] or tree [1. It appears that in our case we can extend the integral Bailey chain described in Theorem 1 to a tree due to a complimentary integral Bailey lemma described below.

Theorem 2. Whenever two functions $\alpha(z, t)$ and $\beta(z, t)$ form an integral elliptic Bailey pair with respect to $t,|t|<1$, the new functions defined for $w \in \mathbb{T}$ as

$$
\alpha^{\prime}(w, t)=\kappa \frac{\Gamma\left(s^{2} t^{2}, u w^{ \pm}\right)}{\Gamma\left(s^{2}, t^{2}, w^{ \pm 2}, t^{2} s^{2} u w^{ \pm}\right)} \int_{\mathbb{T}} \frac{\Gamma\left(t^{2} s u x^{ \pm}, s w^{ \pm} x^{ \pm}\right)}{\Gamma\left(s u x^{ \pm}\right)} \alpha(x, s t) \frac{d x}{x}
$$

and

$$
\beta^{\prime}(w, t)=\frac{\Gamma\left(t u w^{ \pm}\right)}{\Gamma\left(t s^{2} u w^{ \pm}\right)} \beta(w, s t)
$$

form an integral elliptic Bailey pair with respect to $t$ as well. Here $s$ and $u$ are arbitrary complex parameters (they are not related to $s, u$ appearing in Theorem 1) satisfying the constraints $|s|,|u|<1,|p q|<\left|t^{2} s^{2} u\right|$.

Proof. Verification of this statement follows the proof of the previous theorem. For this it is necessary to replace $\alpha(w, t)$ and $\beta(w, t)$ in equality (4) by primed expressions, to substitute relation (15) into it, to change the order of integrations, and to apply formula (2). We skip the details of consideration for their simplicity. 
In [3, only one Bailey lemma was established for elliptic hypergeometric series. Our Theorem 1 was derived in a heuristic analogy with that. It is natural to expect that there exists a limiting process which would allow one to pass from Theorem 1 to the corresponding Bailey chain for series. Evidently, our Theorem 2 should have a series analogue as well which would lead to an elliptic Bailey tree for series (its analogue for $q$-hypergeometric series would be qualitatively different from the tree considered in 1, 4).

As an illustration, we describe one particular sequence of identities generated by the derived Bailey tree. For this we take functions (9) and (10) and apply to them transformations (15) and (16) with parameters $s=s_{1}, u=u_{1}$. Then we apply to the resulting $\alpha^{\prime}(w, t)$ and $\beta^{\prime}(w, t)$ transformations (5) and (6) with the parameters $s=s_{2}, u=u_{2}$. As a result, we obtain

$$
\begin{aligned}
\alpha^{\prime \prime}\left(w, s_{2} t\right)= & \kappa \frac{\Gamma\left(t^{2} s_{1}^{2}, t u_{2} w^{ \pm}, u_{1} w^{ \pm}\right)}{\Gamma\left(s_{1}^{2}, t^{2}, w^{ \pm 2}, t s_{2}^{2} u_{2} w^{ \pm}, t^{2} s_{1}^{2} u_{1} w^{ \pm}\right)} \\
& \times \int_{\mathbb{T}} \frac{\Gamma\left(t^{2} s_{1} u_{1} x^{ \pm}, s_{1} w^{ \pm} x^{ \pm}\right) \prod_{r=0}^{2} \Gamma\left(t_{r} x^{ \pm}\right)}{\Gamma\left(x^{ \pm 2}, s_{1} u_{1} x^{ \pm}, t^{2} s_{1}^{2} t_{0} t_{1} t_{2} x^{ \pm}\right)} \frac{d x}{x} \\
\beta^{\prime \prime}\left(w, s_{2} t\right)= & \kappa \frac{\Gamma\left(t^{2} s_{2}^{2}, t^{2} s_{1}^{2}, t^{2} s_{2} u_{2} w^{ \pm}\right)}{\Gamma\left(s_{2}^{2}, t^{2}, s_{2} u_{2} w^{ \pm}\right)} \prod_{0 \leq r<j \leq 2} \frac{\Gamma\left(t_{r} t_{j}\right)}{\Gamma\left(t^{2} s_{1}^{2} t_{r} t_{j}\right)} \\
& \times \int_{\mathbb{T}} \frac{\Gamma\left(s_{2} w^{ \pm} x^{ \pm}, t u_{1} x^{ \pm}, u_{2} x^{ \pm}\right) \prod_{r=0}^{2} \Gamma\left(t s_{1} t_{r} x^{ \pm}\right)}{\Gamma\left(x^{ \pm 2}, t^{2} s_{2}^{2} u_{2} x^{ \pm}, t s_{1}^{2} u_{1} x^{ \pm}, t s_{1} t_{0} t_{1} t_{2} x^{ \pm}\right)} \frac{d x}{x} .
\end{aligned}
$$

Substituting these functions into basic relation (4) with $t$ replaced by $s_{2} t$, we obtain an equality for two integrals

$$
\begin{aligned}
\int_{\mathbb{T}} \frac{\Gamma\left(s_{2} w^{ \pm} x^{ \pm}, t u_{1} x^{ \pm}, u_{2} x^{ \pm}\right) \prod_{r=0}^{2} \Gamma\left(t s_{1} t_{r} x^{ \pm}\right)}{\Gamma\left(x^{ \pm 2}, t^{2} s_{2}^{2} u_{2} x^{ \pm}, t s_{1}^{2} u_{1} x^{ \pm}, t s_{1} t_{0} t_{1} t_{2} x^{ \pm}\right)} \frac{d x}{x} \\
=\kappa \prod_{0 \leq r<j \leq 2} \frac{\Gamma\left(t^{2} s_{1}^{2} t_{r} t_{j}\right)}{\Gamma\left(t_{r} t_{j}\right)} \frac{\Gamma\left(s_{2}^{2}, s_{2} u_{2} w^{ \pm}\right)}{\Gamma\left(s_{1}^{2}, t^{2} s_{2}^{2}, t^{2} s_{2} u_{2} w^{ \pm}\right)} \\
\quad \times \int_{\mathbb{T}^{2}} \Gamma\left(s_{1} x_{2}^{ \pm} x_{1}^{ \pm}\right) \frac{\Gamma\left(t s_{2} w^{ \pm} x_{2}^{ \pm}, t u_{2} x_{2}^{ \pm}, u_{1} x_{2}^{ \pm}\right)}{\Gamma\left(x_{2}^{ \pm 2}, t s_{2}^{2} u_{2} x_{2}^{ \pm}, t^{2} s_{1}^{2} u_{1} x_{2}^{ \pm}\right)} \\
\times \frac{\Gamma\left(t^{2} s_{1} u_{1} x_{1}^{ \pm}\right) \prod_{r=0}^{2} \Gamma\left(t_{r} x_{1}^{ \pm}\right)}{\Gamma\left(x_{1}^{ \pm 2}, s_{1} u_{1} x_{1}^{ \pm}, t^{2} s_{1}^{2} t_{0} t_{1} t_{2} x_{1}^{ \pm}\right)} \frac{d x_{1}}{x_{1}} \frac{d x_{2}}{x_{2}} .
\end{aligned}
$$

This identity was derived under the constraints that all parameters lie inside the unit circle and $\left|t^{2} s_{1}^{2} t_{0} t_{1} t_{2}\right|,\left|t^{2} s_{1}^{2} u_{2}\right|,\left|t^{2} s_{2}^{2} u_{2}\right|>|p q|$. However, by analyticity it remains true for a larger region of values of parameters provided we replace $\mathbb{T}$ by contours of integration which encircle sequences of poles of the integrands converging to zero and exclude other ones. 
It is not difficult to find the result of $m$-fold iteration of transformations (15) and (16) with different parameters $u_{k}, s_{k}$ :

$$
\begin{aligned}
\alpha^{(m)}\left(x_{m+1}, t\right) & =\kappa^{m} \prod_{k=1}^{m} \frac{\Gamma\left(\prod_{l=k}^{m} s_{l}^{2} t^{2}\right)}{\Gamma\left(s_{k}^{2}, \prod_{l=k+1}^{m} s_{l}^{2} t^{2}\right)} \\
\times \int_{\mathbb{T}^{m}} & \prod_{k=1}^{m} \frac{\Gamma\left(u_{k} x_{k+1}^{ \pm}, \prod_{l=k+1}^{m} s_{l}^{2} t^{2} s_{k} u_{k} x_{k}^{ \pm}, s_{k} x_{k+1}^{ \pm} x_{k}^{ \pm}\right)}{\Gamma\left(x_{k+1}^{ \pm 2}, \prod_{l=k}^{m} s_{l}^{2} t^{2} u_{k} x_{k+1}^{ \pm}, s_{k} u_{k} x_{k}^{ \pm}\right)} \\
& \times \alpha^{(0)}\left(x_{1}, \prod_{l=1}^{m} s_{l} t\right) \frac{d x_{1}}{x_{1}} \cdots \frac{d x_{m}}{x_{m}}, \\
\beta^{(m)}(w, t)= & \prod_{k=1}^{m} \frac{\Gamma\left(t \prod_{l=k+1}^{m} s_{l} u_{k} w^{ \pm}\right)}{\Gamma\left(t \prod_{l=k+1}^{m} s_{l} s_{k}^{2} u_{k} w^{ \pm}\right)} \beta^{(0)}\left(w, \prod_{l=1}^{m} s_{l} t\right) .
\end{aligned}
$$

An application of transformations (515) and (6) with the parameters $s=s_{m+1}, u=$ $u_{m+1}$ to these functions yields:

$$
\begin{aligned}
& \alpha^{\prime}\left(x_{m+1}, s_{m+1} t\right)=\frac{\Gamma\left(t u_{m+1} x_{m+1}^{ \pm}\right)}{\Gamma\left(t s_{m+1}^{2} u_{m+1} x_{m+1}^{ \pm}\right)} \alpha^{(m)}\left(x_{m+1}, t\right), \\
& \beta^{\prime}\left(w, s_{m+1} t\right)=\kappa \frac{\Gamma\left(t^{2} s_{m+1}^{2}, t^{2} s_{m+1} u_{m+1} w^{ \pm}\right)}{\Gamma\left(s_{m+1}^{2}, t^{2}, s_{m+1} u_{m+1} w^{ \pm}\right)} \\
& \times \int_{\mathbb{T}} \frac{\Gamma\left(s_{m+1} w^{ \pm} x^{ \pm}, u_{m+1} x^{ \pm}\right)}{\Gamma\left(x^{ \pm 2}, t^{2} s_{m+1}^{2} u_{m+1} x^{ \pm}\right)} \beta^{(m)}(x, t) \frac{d x}{x} \\
& =\kappa \int_{\mathbb{T}} \Gamma\left(s_{m+1} t w^{ \pm} x_{m+1}^{ \pm}\right) \alpha^{\prime}\left(x_{m+1}, s_{m+1} t\right) \frac{d x_{m+1}}{x_{m+1}} .
\end{aligned}
$$

We substitute into the latter relation expressions (20) and (21) with functions (9) and (10) serving as $\alpha^{(0)}(w, t)$ and $\beta^{(0)}(w, t)$. As a result, we derive the identity

$$
\begin{aligned}
\int_{\mathbb{T}} \frac{\Gamma\left(s_{m+1} w^{ \pm} x^{ \pm}, u_{m+1} x^{ \pm}\right)}{\Gamma\left(x^{ \pm 2}, t^{2} s_{m+1}^{2} u_{m+1} x^{ \pm}\right)} \prod_{k=1}^{m} \frac{\Gamma\left(t \prod_{l=k+1}^{m} s_{l} u_{k} x^{ \pm}\right)}{\Gamma\left(t \prod_{l=k+1}^{m} s_{l} s_{k}^{2} u_{k} x^{ \pm}\right)} \\
\quad \times \frac{\prod_{r=0}^{2} \Gamma\left(t \prod_{k=1}^{m} s_{k} t_{r} x^{ \pm}\right)}{\Gamma\left(t \prod_{k=1}^{m} s_{k} t_{0} t_{1} t_{2} x^{ \pm}\right)} \frac{d x}{x}=\frac{\kappa^{m} \Gamma\left(s_{m+1}^{2}, t^{2}, s_{m+1} u_{m+1} w^{ \pm}\right)}{\Gamma\left(t^{2} s_{m+1}^{2}, t^{2} \prod_{k=1}^{m} s_{k}^{2}, t^{2} s_{m+1} u_{m+1} w^{ \pm}\right)} \\
\quad \times \prod_{k=1}^{m} \frac{\Gamma\left(\prod_{l=k}^{m} s_{l}^{2} t^{2}\right)}{\Gamma\left(s_{k}^{2}, \prod_{l=k+1}^{m} s_{l}^{2} t^{2}\right)} \prod_{0 \leq r<j \leq 2} \frac{\Gamma\left(t^{2} \prod_{k=1}^{m} s_{k}^{2} t_{r} t_{j}\right)}{\Gamma\left(t_{r} t_{j}\right)} \\
\quad \times \int_{\mathbb{T}^{m+1}} \frac{\Gamma\left(t s_{m+1} w^{ \pm} x_{m+1}^{ \pm}, t u_{m+1} x_{m+1}^{ \pm}\right) \prod_{r=0}^{2} \Gamma\left(t_{r} x_{1}^{ \pm}\right)}{\Gamma\left(t s_{m+1}^{2} u_{m+1} x_{m+1}^{ \pm}, x_{1}^{ \pm 2}, t^{2} \prod_{k=1}^{m} s_{k}^{2} t_{0} t_{1} t_{2} x_{1}^{ \pm}\right)} \\
\quad \times \prod_{k=1}^{m} \frac{\Gamma\left(u_{k} x_{k+1}^{ \pm}, \prod_{l=k+1}^{m} s_{l}^{2} t^{2} s_{k} u_{k} x_{k}^{ \pm}, s_{k} x_{k+1}^{ \pm} x_{k}^{ \pm}\right)}{\Gamma\left(x_{k+1}^{ \pm 2}, \prod_{l=k}^{m} s_{l}^{2} t^{2} u_{k} x_{k+1}^{ \pm}, s_{k} u_{k} x_{k}^{ \pm}\right)} \frac{d x_{1}}{x_{1}} \cdots \frac{d x_{m+1}}{x_{m+1}} .
\end{aligned}
$$

For $m=1$ this equality is reduced to relation (19).

Evidently, a more thorough analysis of consequences of the derived elliptic Bailey tree for integrals with different multiplicities of integrations is desirable. In particular, uniqueness of this tree is an interesting question for consideration. Implications for $q$-hypergeometric integrals and elliptic and $q$-hypergeometric series are worth of detailed investigation as well. 
This work is supported in part by the Russian Foundation for Basic Research (RFBR) Grant No. 03-01-00780.

\section{REFERENCES}

[1] G.E. Andrews, Bailey's transform, lemma, chains and tree, Proc. NATO ASI Special functions-2000 (Tempe, USA, May 29-June 9, 2000), Kluwer, Dordrecht, 2001, pp. 1-22.

[2] S.O. Warnaar, 50 years of Bailey's lemma, Algebraic Combinatorics and Applications (Springer, Berlin, 2001), pp. 333-347.

[3] V.P. Spiridonov, An elliptic incarnation of the Bailey chain, Internat. Math. Res. Notices, no. 37 (2002), 1945-1977.

[4] G.E. Andrews and A. Berkovich, The WP-Bailey tree and its implications, J. London Math. Soc (2) 66 (2002), 529-549.

[5] V.P. Spiridonov, Theta hypergeometric integrals, Algebra i Analiz (St. Petersburg Math. J.) 15 (6) (2003), 161-215.

[6] V.P. Spiridonov, An elliptic beta integral, Proc. Fifth Internat. Conf. on Difference Equations (Temuco, Chile, January 3-7, 2000), Taylor and Francis, London, 2001, pp. 273282; On the elliptic beta function, Russ. Math. Surveys 56 (1) (2001), 185-186.

[7] G. Gasper and M. Rahman, Basic Hypergeometric Series, Encyclopedia of Mathematics and its Applications 35, Cambridge Univ. Press, Cambridge, 1990.

[8] G.E. Andrews, Multiple series Rogers-Ramanujan type identities, Pacific J. Math. 114 (1984), 267-283.

[9] K. Garrett, M.E.H. Ismail, and D. Stanton, Variants of the Rogers-Ramanujan identities, Adv. Appl. Math. 23 (1999), 274-299.

[10] G. Anderson, A short proof of Selberg's generalized beta formula, Forum Math. 3 (1991), 415-417.

[11] R.Y. Denis and R.A. Gustafson, An $S U(n)$ q-beta integral transformation and multiple hypergeometric series identities, SIAM J. Math. Anal. 23 (1992), 552-561.

[12] R.A. Gustafson, Some q-beta integrals on $S U(n)$ and $S p(n)$ that generalize the AskeyWilson and Nassrallah-Rahman integrals, SIAM J. Math. Anal. 25 (1994), 441-449.

[13] R.A. Gustafson and M.A. Rakha, q-Beta integrals and multivariate basic hypergeometric series associated to root systems of type $A_{m}$, Ann. Comb. 4 (2000), 347-373.

[14] J.F. van Diejen and V.P. Spiridonov, Elliptic Selberg integrals, Internat. Math. Res. Notices, no. 20 (2001), 1083-1110.

[15] G.E. Andrews, R. Askey, and R. Roy, Special Functions, Encyclopedia of Mathematics and its Applications 71, Cambridge Univ. Press, Cambridge, 1999.

[16] A.K. Agarwal, G.E. Andrews, and D.M. Bressoud, The Bailey lattice, J. Indian Math. Soc. (N.S.) 51 (1987), 57-73.

Bogoliubov Laboratory of Theoretical Physics, JinR, Dubna, Moscow Reg. 141980, RUSSIA 